\title{
Throughput performance for full-duplex DF relaying protocol in hybrid wireless power transfer systems
}

\author{
Hoang-Phuong Van, Hoang-Sy Nguyen \\ Institute of Engineering and Technology, Thu Dau Mot University, Binh Duong Province, Vietnam
}

\begin{tabular}{l} 
Article Info \\
\hline Article history: \\
Received Apr 28, 2021 \\
Revised Oct 29, 2021 \\
Accepted Nov 3, 2021 \\
\hline
\end{tabular}

\section{Keywords:}

Full-duplex DF relaying

Hybrid TS-PS

Log-normal fading

Outage probability

Wireless power transfer

\begin{abstract}
Most of the existing studies on energy harvesting (EH) cooperative relaying networks are conducted for the outdoor environments which are mainly characterized by Rayleigh fading channels. However, there are not as many studies that consider the indoor environments whereas the state-of-the-art internet of things (IoT) and smart city applications are built upon. Thus, in this paper, we analyze a namely hybrid time-power splitting relaying (HTPSR) protocol in a full-duplex (FD) decode-and-forward (DF) batteryenergized relaying network in indoor scenarios modelled by the unpopular log-normal fading channels. Firstly, we formulate the analytical expression of the outage probability (OP) then the system throughput. Accordingly, we simulate the derived expressions with the Monte Carlo method. It is worth mentioning that in our work, the simulation and the theory agree well with each other. From the simulation results, we know how to compromise either the power splitting (PS) or the time splitting (TS) factors for optimizing the system performance.
\end{abstract}

This is an open access article under the CC BY-SA license.

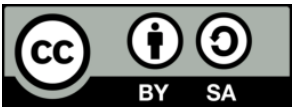

\section{Corresponding Author:}

Hoang-Sy Nguyen

Institute of Engineering and Technology

Thu Dau Mot University

Binh Duong Province, Vietnam

Email: nguyenhoangsy@tdmu.edu.vn

\section{INTRODUCTION}

Finite-capacity batteries that we use to power cooperative relays of wireless networks have some downside of being difficult or even impractical to replace or recharge [1]-[3]. Thus, we can consider renewable resources as promising alternatives, [4]-[6] even though they cannot be deployed for highly reliable systems because of their unstable nature [7]. As a better solution, we can exploit a so-called simultaneous information and power transfer (SWIPT) technology developed for energy harvesting (EH) from radio frequency (RF) signals, which was investigated, [8]-[10]. The primary idea behind the SWIPT system is that a part of the same RF signal that is ordinarily used for the information transmission between sources and destinations can be harvested by thein-between relays, which then can be stored or directly utilized in the networks to self-sustain their operation. As a matter of fact, such EH cooperative relaying wireless networks has been studied extensively and their system performance is primarily evaluated in terms of the outage probability (OP) and the throughput of the systems [11]-[19]. In particular, the OP of powersplitting (PS) SWIPT networks was investigated with [11] and with-out [12]-[14] the presence of the direct link. Employing Markov model, Li et al. [15] considered the status of batteries in SWIPT networks before attempting to minimize the OP. In the context of power time splitting-based (PTS) amplify-and-forward (AF) relaying networks, it was reported in [16] that the relay position plays a significant role in determining the system performance. Later, Ojo and Salleh [17] proposed a namely hybrid power-time splitting based 
relaying protocol (HPTSR) and analyzed its throughput performance in AF and decode-and-forward (DF) scenarios. The throughput of two-way AF relaying networks in three different schemes namely two, three, four-time-slot (2TS, 3TS, 4TS) was investigated in [18]. Khan et al. [19], one of the most recent studies, two relay selection schemes so-called joint relay power allocation and selection scheme (JPASS) and ratio selection scheme (RSS) were proposed and proven with remarkably better average sum-throughput in comparison with conventional schemes.

We can find several studies of SWIPT systems in outdoor scenarios where authors employed Rayleigh, Nakagami-m and Rician models for characterizing the fading effects [20], [21]. On the other hand, studies concerning the indoor scenarios using the log-normal model are rare. As proven in some old studies, we could use the model to simulate the fading variation indoor due to the moving objects and walls, [22]-[24]. Specifically, for indoor scenarios, we can expect that employing cooperative relays will make the system perform better. Indeed, few recent studies concerning the indoor log-normal fading proved this point [25]-[28], which sheds light on the implementation of such channel in the internet of things (IoT). Specifically, as for an indoor IoT network, the communication between in-house devices would subject to the shadowing effect that can be best modeled with log-normal fading. Moreover, due to the continuous growth of the relaying protocols utilized for the future $5 \mathrm{G}$ devices, beside the conventional relaying protocols, assessing a hybrid scheme such as the hybrid time-power splitting relaying (HTPSR) is of importance. Inspired by the above studies, herein, we evaluate the performance of the HTPSR scheme by means of the throughput over lognormal fading channels in the context of an indoor full-duplex (FD) decode-and-forward (DF) relaying network. In particular, the throughput and its relationship with the two primary factors of the HTPSR scheme was analytically expressed. Then, based on the expression, Monte Carlo simulations were done in MATLAB and the results obtained were compared with the theory.

After this introduction, readers can find the system model in section 2. Section 3 analyzes the performance of the HTPSR protocol and the whole system in terms of the OP. Moreover, there are Monte Carlo simulation results and discussion in section 4. Finally, section 5 is used to conclude the findings of this paper.

\section{SYSTEM MODEL}

We demonstrate in Figure 1 a dual-hop network which consists of one source (S), one destination (D) and one relay (R). The communication is possible only via $\mathrm{S}$ to $\mathrm{R}$, then $\mathrm{R}$ to $\mathrm{D}$. We denote the two distances with $d_{S R}$ and $d_{R D}$, and assign them with channel coefficients $d_{S R}$ and $d_{R D}$, respectively. R operates in FD mode and subject to a loop interference of $h_{L I}$. This configuration is generally used for log-normal fading channel studies, [25], [27]. Additionally, R is energized entirely with the EH module which collects the energy from the signal that $\mathrm{S}$ transmits. Moreover, we have the path-loss exponent denoted as $m$.

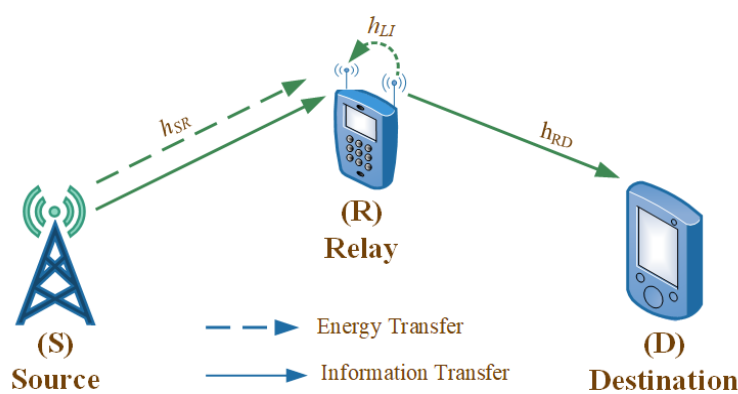

Figure 1. System model

Besides, we have $\left|h_{S R}\right|^{2}$ and $\left|h_{R D}\right|^{2}$ as two independent and identically distributed (i.i.d) log-normal random variables (RVs) and we specify them with parameters $L N\left(\mu_{S R}, \sigma_{S R}^{2}\right)$ and $L N\left(\mu_{R D}, \sigma_{R D}^{2}\right)$, respectively. It should be noted that the $\mu_{i}$ and $\sigma_{i}^{2}$ are the mean and the standard deviation of $10 \log \left(\left|h_{i}\right|^{2}\right), i \in\{S R, R D\}$ and the unit of both are $(\mathrm{dB})$. Moreover, we denote the loop interference channel with $\left|h_{L I}\right|^{2}$ and lognormally distribute it with parameters $L N\left(\mu_{L I}, \sigma_{L I}^{2}\right)$. Indeed, this parameter determines the strength of the loop interference, thus, is essential for the overall system performance.

Then, we can formulate the probability density function (PDF) and cumulative distribution function (CDF) of log-normally distributed RV $\left|h_{i}\right|^{2}$, respectively as: 


$$
f_{\left|h_{i}\right|^{2}}(x)=\frac{\xi}{x \sigma_{i} \sqrt{2 \pi}} \exp \left(-\frac{1}{2} \frac{\left(\xi \ln (x)-\mu_{i}\right)^{2}}{\sigma_{i}^{2}}\right)
$$

and

$$
F_{\left|h_{i}\right|^{2}}(x)=1-\mathcal{Q}\left(\frac{\xi \ln (x)-2 \mu_{i}}{2 \sigma_{i}}\right),
$$

where we have the scaling constant $\xi=\frac{10}{\ln (10)}$, and Gaussian Q-function $\mathcal{Q}(x)=\int_{x}^{\infty} \frac{1}{\sqrt{2 \pi}} \exp \left(-\frac{t^{2}}{2}\right) d t$.

We depict in Figure 2 the HTPSR protocol for log-normal systems where we partition the transmission time block $T$ to two slots, $\alpha T$ and $(1-\alpha) T$, with TS factor $\alpha,(\alpha \in[0,1])$. In the 1 st time slot $\alpha T, \mathrm{R}$ allocates $\sqrt{\beta}$ for $\mathrm{EH}$ and $\sqrt{1-\beta}$ to receive the information from $\mathrm{S}$. In the 2nd time slot $(1-\alpha) T$, all the harvested energy is used for decoding and forwarding the signal from S to D. We can neglect the relay's processing power since it is relatively small compared with the transmission power from $\mathrm{R}$ to $\mathrm{D}$.

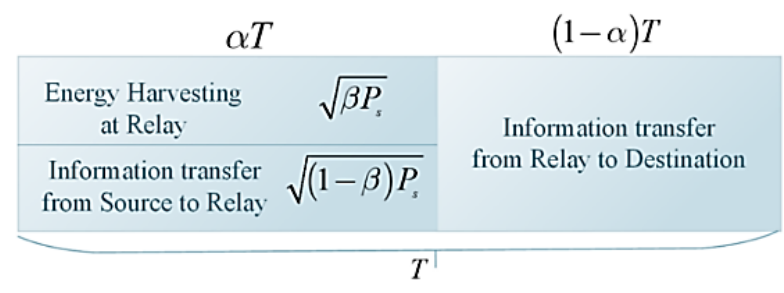

Figure 2. HPTSR protocol for log-normal fading channel

\section{PERFORMANCE ANALYSIS OF THE HTPSR PROTOCOL}

In the $1^{\text {st }}$ time slot, $\mathrm{R}$ harvests the energy amount of (3),

$$
E=\eta \beta T \frac{\alpha P_{S}\left|h_{S R}\right|^{2}}{d_{S R}^{m}}
$$

where we denote the energy conversion efficiency as $\eta,(\eta \in(0,1))$. Then, we can obtain the base-band signal at $\mathrm{R}$ in FD mode, $\mathrm{y}_{\mathrm{SR}}$, during the EH phase of $\sqrt{(1-\beta)}$ from:

$$
y_{S R}=\sqrt{\frac{(1-\beta) P_{S}}{d_{1}^{m}}} h_{S R} x_{S}+\sqrt{(1-\beta) P_{R}} h_{L I} x_{r}+n_{r}
$$

where we have the information signal $\mathrm{x}_{\mathrm{S}}$, and the loop interference $\mathrm{x}_{\mathrm{r}}$, normalized respectively with $\mathbb{E}\left[\left|\mathrm{x}_{\mathrm{s}}\right|\right]^{2}=1$, and $\mathbb{E}\left[\left|\mathrm{x}_{\mathrm{r}}\right|\right]^{2}=1$. The narrow-band Gaussian noise $\mathrm{n}_{\mathrm{r}}$, with zero mean and variance $\mathrm{N}_{0}$, is caused by R's receiving antenna.

Since the FD R can recognize the signal of itself, it can apply the interference cancellation process to minimize its loop interference. Hence, we can obtain the signal at R, after cancellation as (5).

$$
y_{r}=\sqrt{\frac{(1-\beta) P_{S}}{d_{1}^{m}}} h_{S R} x_{s}+\sqrt{(1-\beta) P_{R}} \bar{h}_{L I} \bar{x}_{r}+n_{r}
$$

Where $\bar{h}_{L I}$ is the residual loop interference channel owing to the imperfect interference cancellation and $\mathbb{E}\left[\left|\bar{x}_{r}\right|\right]^{2}=1$. Under DF relaying protocol, $\mathrm{R}$ will decode and forward the signal from $\mathrm{S}$ to $\mathrm{D}$. Hence, $\mathrm{D}$ receives signal of $(6)$,

$$
y_{d}=\sqrt{\frac{P_{R}}{d_{R D}^{m}}} h_{R D} x_{s}+n_{d}
$$

where the narrow-band Gaussian noise at D node $n_{d}$, with zero mean and variance $N_{0}$.

Besides, $\mathrm{R}$ transmits power to $\mathrm{D}$ in the 2 nd time slot, $(1-\alpha) T$. This power is expressed as (7).

$$
P_{R}=\frac{E}{(1-\alpha) T}=\frac{\eta \alpha \beta}{(1-\alpha)} \frac{\left|h_{S R}\right|^{2}}{d_{S R}^{m}} P_{S} .
$$


Now, we analytically express important metrics for the system performance. By substituting (4) into (5) and (7), we can obtain the signal-to-noise ratio (SNR) at R denoted as $\gamma_{S R}$, and D as $\gamma_{R D}$, in the HTPSR scheme, respectively, as:

$$
\gamma_{S R} \approx \frac{(1-\beta) P_{S}\left|h_{S R}\right|^{2}}{(1-\beta) P_{R}\left|h_{L I}\right|^{2} d_{S R}^{m}}=\frac{(1-\alpha)}{\eta \alpha \beta\left|h_{L I}\right|^{2}}
$$

and

$$
\gamma_{R D}=\frac{\eta \alpha \beta P_{S}\left|h_{S R}\right|^{2}\left|h_{R D}\right|^{2}}{(1-\beta) d_{S R}^{m} d_{R D}^{m} N_{0}}
$$

With regard to (8) and (9), we can formulate the achievable data rate at R and D, respectively, as:

$$
R_{S R}=W \alpha \log _{2}\left(1+\frac{(1-\alpha)}{\eta \alpha \beta\left|h_{L I}\right|^{2}}\right),
$$

and

$$
R_{R D}=W(1-\alpha) \log _{2}\left(1+\frac{\eta \alpha \beta P_{S}\left|h_{S R}\right|^{2}\left|h_{R D}\right|^{2}}{(1-\beta) d_{S R}^{m} d_{R D}^{m} N_{0}}\right)
$$

where $W$ stands for the bandwidth system.

The outage probability, $P_{\text {out }}$ at the D node is defined as the probability that the achievable data rate at $\mathrm{R}$ and $\mathrm{D}$ descends below the target data rate. Thus, we have $P_{\text {out }}=\operatorname{Pr}\left(\min \left(R_{\mathrm{SR}}, R_{\mathrm{RD}}\right)<R_{0}\right)$, where $R_{0}=\log _{2}\left(1+\gamma_{0}\right)$, with the predetermined threshold value of SNR, $\gamma_{0}$. Indeed, the $\left|h_{S R}\right|^{2},\left|h_{R D}\right|^{2}$ and $\left|h_{L I}\right|^{2}$ are three independent random variables (RVs). Exploiting the log-normal distribution property, we can formulate the OP as:

$$
P_{\text {out }}=1-\underbrace{\bar{F}_{\left|h_{L I}\right|^{2}}\left(\frac{\eta \alpha \beta}{1-\alpha}\left(2^{\frac{R_{0}}{\alpha W}}-1\right)\right)}_{P_{1}} \times \underbrace{\bar{F}_{\left|h_{S R}\right|^{2}\left|h_{R D}\right|^{2}}\left(\frac{(1-\beta) d_{S R}^{m} d_{R D}^{m} N_{0}}{\eta \alpha \beta P_{S}}\left(2^{\frac{R_{0}}{(1-\alpha) W}}-1\right)\right)}_{P_{2}},
$$

where $\bar{F}_{X}($.$) is the complementary cumulative distribution function (CCDF) of RV X$.

The first part, $P_{1}$ in (12) can be obtained from:

$$
P_{1}=\mathcal{Q}\left(\frac{\xi \ln \left(\kappa_{1} v_{1}\right)-2 \mu_{L I}}{2 \sigma_{L I}}\right)
$$

where $\kappa_{1}=\frac{\eta \alpha \beta}{1-\alpha}$, and $v_{1}=2^{\frac{R_{0}}{\alpha W}}-1$. The second part, $P_{2}$ in (12) can be acquired from.

$$
P_{2}=\mathcal{Q}\left(\frac{\xi \ln \left(\kappa_{2} v_{2}\right)-2 \mu_{S R}-2 \mu_{R D}}{\sqrt{2} \sigma_{S R}+\sqrt{2} \sigma_{S R}}\right),
$$

where $\kappa_{2}=\frac{(1-\beta) d_{S R}^{m} d_{R D}^{m} N_{0}}{\eta \alpha \beta P_{S}}$, and $v_{2}=2^{\frac{R_{0}}{(1-\alpha) W}}-1$. We substitute (13) and (14) into (12) to attain the $P_{\text {out }}$ at D as:

$$
P_{\mathrm{out}}=1-\mathcal{Q}\left(\frac{\xi \ln \left(\kappa_{1} v_{1}\right)-2 \mu_{L I}}{2 \sigma_{L I}}\right) \times \mathcal{Q}\left(\frac{\xi \ln \left(\kappa_{2} v_{2}\right)-2 \mu_{S R}-2 \mu_{R D}}{\sqrt{2} \sigma_{S R}+\sqrt{2} \sigma_{S R}}\right) .
$$

We can formulate the throughput at the $\mathrm{D}$ node with a fixed source transmission rate, $R_{0}$ ( $\mathrm{bits} / \mathrm{sec} / \mathrm{Hz}$ ), and the effective communication time $(1-\alpha) T$ over the time block $T$ (second) for the HTPSR scheme as (16),

$\tau=R_{0}\left(1-P_{\text {out }}\right) \frac{(1-\alpha) T}{T}=R_{0}(1-\alpha)\left(1-P_{\text {out }}\right)$,

with formulated $P_{\text {out }}$ in (15). 


\section{RESULT AND DISCUSSION}

We analyze the impact of EH TS and EH PS factors on the throughput of the HTPSR system thanks to the Monte Carlo simulation results. In the Table 1 we list out the parameters that were used. Figure 3 and Figure 4 illustrate the throughput versus, respectively, the EH TS and EH PS factor. We plotted the curves with three different power transmission levels and with either of the EH factors fixed at 0.3. It is clear that the higher the power transmission, $\mathrm{P}_{\mathrm{S}}$, the better the throughput value. As for Figure 3, if we fix the EH PS factor at 0.3 and vary the EH TS factor, the throughput for the three curves peaks at EH TS factor of about 0.25.

Table 1. The main parameters

\begin{tabular}{cccc}
\hline Parameters & Values & Parameters & Values \\
\hline $\mathrm{W}$ & $5(\mathrm{~dB})$ & $\mathrm{P}_{\mathrm{S}}$ & $1(\mathrm{~dB})$ \\
$\eta$ & $80 \%$ & $\sigma_{\mathrm{LI}}^{2}$ & $3(\mathrm{~dB})$ \\
$\mathrm{m}$ & 3 & $\mu_{\mathrm{LI}}$ & $2.5(\mathrm{~dB})$ \\
$\sigma_{\mathrm{SR}}^{2}$ & $3(\mathrm{~dB})$ & $\mathrm{d}_{\mathrm{SR}}$ & $2(\mathrm{~m})$ \\
$\mu_{\mathrm{SR}}$ & $4(\mathrm{~dB})$ & $\mathrm{d}_{\mathrm{SR}}$ & $2(\mathrm{~m})$ \\
$\mu_{\mathrm{RS}}$ & $4(\mathrm{~dB})$ & $\sigma_{\mathrm{RS}}^{2}$ & $3(\mathrm{~dB})$ \\
\hline
\end{tabular}

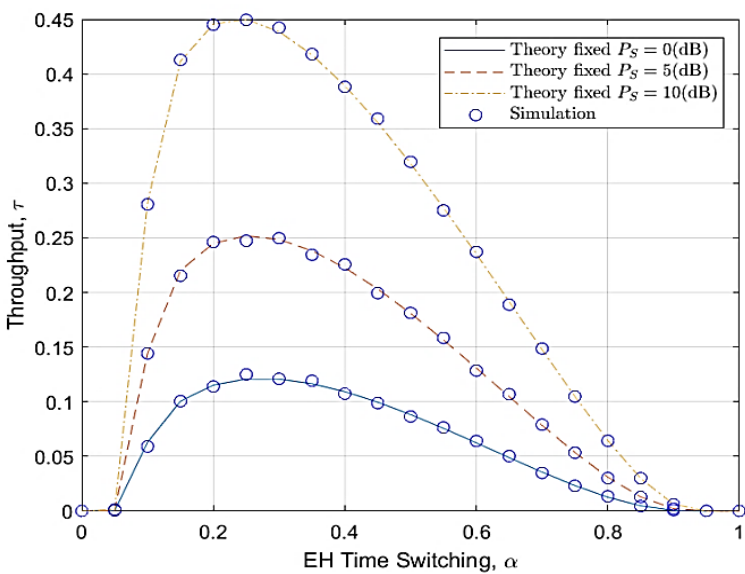

Figure 3. Throughput versus EH TS factor with various power transmission and EH PS fixed at 0.3

Besides, for Figure 4, as we fix the EH TS factor at 0.3 and vary the EH PS factor, the throughput of the three curves peaks at EH PS factor around 0.95 then sharply drops to 0 . Another obvious trait that we can see from the two figures is that the throughput level starts from and ends at 0 as the EH TS and EH PS factors start from 0 or approach 1 . Moreover, it is worth noting that the simulation results correlate well with the theoretical ones.

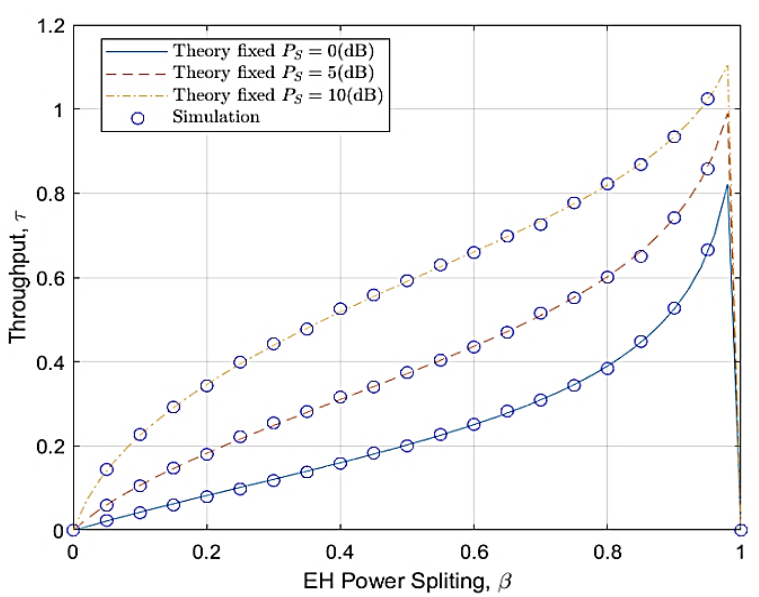

Figure 4. Throughput versus EH PS factor with various power transmission and EH TS fixed at 0.3 
Figure 5 depicts the throughput versus the SNR. We can observe that the throughput is directly proportional to the SNR. The throughput curve is the best, the highest, at EH TS=0.3 and EH PS=0.5 As we decrease the EH PS to 0.3, the throughput curve decreases. The throughput curve is the worst, the lowest, as we increase the EH TS to 0.5 while keeping the EH PS at 0.3 . The simulation results align well with the theoretical curves. This proves that the previously derived expressions are relatively accurate and can be used for future studies.

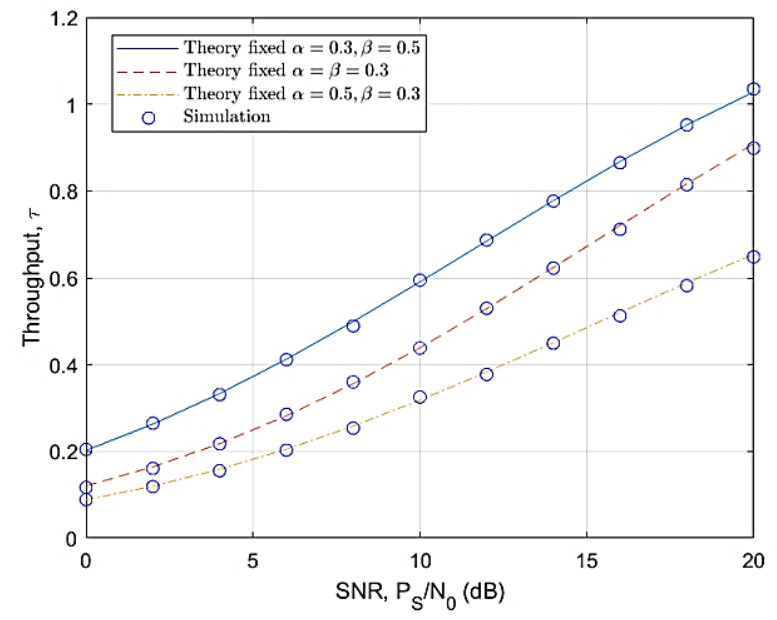

Figure 5. Throughput versus SNR with various pairs of EH TS and EH PS factors

\section{CONCLUSION}

In a nutshell, we investigate the HTPSR protocol in an EH DF relaying network for indoor scenario characterized by log-normal fading channels in terms of the system throughput. Based on the theoretical and simulation results, we understand better the system behavior of the hybrid protocol and how to compromise the PS and TS factors to optimize the system performance. Thanks to the fact that the simulation and the theoretical results correlate well with each other, ones can replicate the expressions derived in this study with some predetermined initial optimal values and further develop it to design indoor IoT networks.

\section{REFERENCES}

[1] D. Shaviv, P. Nguyen, and A. Özgür, "Capacity of the energy-harvesting channel with a finite battery," IEEE Transactions on Information Theory, vol. 62, no. 11, pp. 6436-6458, 2016, doi: 10.1109/TIT.2016.2602353.

[2] S. Narayanan, M. Shikh-Bahaei, J. Hou, and M. F. Flanagan, "Wireless-Powered Distributed Spatial Modulation with Energy Recycling and Finite-Energy Storage," IEEE Transactions on Wireless Communications, vol. 17, no. 10, pp. 6645-6662, Oct. 2018, doi: 10.1109/TWC.2018.2861870.

[3] I. Alhamrouni, M. Iskandar, M. Salem, L. J. Awalin, A. Jusoh, and T. Sutikno, "Application of inductive coupling for wireless power transfer," International Journal of Power Electronics and Drive Systems (IJPEDS), vol. 11, no. 3, pp. 1109-1116, 2020, doi: 10.11591/ijpeds.v11.i3.pp1109-1116.

[4] C. Wang, J. Li, Y. Yang, and F. Ye, "Combining solar energy harvesting with wireless charging for hybrid wireless sensor networks," IEEE Transactions on Mobile Computing, vol. 17, no. 3, pp. 560-576, March 2018, doi: 10.1109/TMC.2017.2732979.

[5] J. Bito, R. Bahr, J. G. Hester, S. A. Nauroze, A. Georgiadis, and M. M. Tentzeris, "A novel solar and electromagnetic energy harvesting system with a 3-D printed package for energy efficient internet-of-things wireless sensors," IEEE Transactions on Microwave Theory and Techniques, vol. 65, no. 5, pp. 1831-1842, May 2017, doi: 10.1109/TMTT.2017.2660487.

[6] M. Shirvanimoghaddam et al., "Towards a green and self-powered internet of things using piezoelectric energy harvesting," IEEE Access, vol. 7, pp. 94533-94556, 2019, doi: 10.1109/ACCESS.2019.2928523.

[7] A. A. Babayo, M. H. Anisi, and I. Ali, "A Review on energy management schemes in energy harvesting wireless sensor networks," Renewable and Sustainable Energy Reviews, vol. 76, pp. 1176-1184, Sep. 2017, doi: 10.1016/j.rser.2017.03.124.

[8] X. Lu, P. Wang, D. Niyato, D. I. Kim, and Z. Han, "Wireless charging technologies: fundamentals, standards, and network applications," IEEE Communications Surveys \& Tutorials, vol. 18, no. 2, pp. 1413-1452, 2016, doi: 10.1109/COMST.2015.2499783. 
[9] X. Liu, F. Li, and Z. Na, "Optimal resource allocation in simultaneous cooperative spectrum sensing and energy harvesting for multichannel cognitive radio," IEEE Access, vol. 5, pp. 3801-3812, 2017, doi: 10.1109/ACCESS.2017.2677976.

[10] H. T. Van, V. T. Anh, D. H. Le, M. Q. Phu, and H.-S. Nguyen, "Outage performance analysis of non-orthogonal multiple access systems with RF energy harvesting," International Journal of Electrical and Computer Engineering (IJECE), vol. 11, no 5, pp. 4135-4142, 2021, doi: 10.11591/ijece.v11i5.pp4135-4142.

[11] H. Lee, C. Song, S. Choi, and I. Lee, "Outage probability analysis and power splitter designs for SWIPT relaying systems with direct link," IEEE Communications Letters, vol. 21, no. 3, pp. 648-651, March 2017, doi: 10.1109/LCOMM.2016.2627055.

[12] F. Wang, W. Xu, S. Li, Z. Feng, and J. Lin, "Outage probability analysis of DF relay networks with RF energy harvesting," in 2015 IEEE Global Communications Conference (GLOBECOM), 2015, pp. 1-5, doi: 10.1109/GLOCOM.2015.7417579.

[13] P. T. Tin, M. Tran, V-D. Phan, H-N. Nguyen, and T. T. Trang, "User selection protocols in FD PSP EH cooperative network over Rayleigh fading channel: Outage and intercept probability," International Journal of Power Electronics and Drive Systems, vol. 10, no. 4, pp. 1109-1116, 2019, doi: 10.11591/ijpeds.v10.i4.2130-2137.

[14] K. Onishi, K. Yamaguchi, and K. Iida, "Wireless power transfer using multiple-transmitters for high stability for position," International Journal of Electrical and Computer Engineering (IJECE), vol. 10, no 3, pp. 2245-2249, June 2020, doi: 10.11591/ijece.v10i3.pp2245-2249.

[15] T. Li, P. Fan, and K. B. Letaief, "Outage probability of energy harvesting relay-aided cooperative networks over rayleigh fading channel," IEEE Transactions on Vehicular Technology, vol. 65, no. 2, pp. 972-978, Feb. 2016, doi: 10.1109/TVT.2015.2402274.

[16] H-S. Nguyen, T-S. Nguyen, V-T. Vo, and M. Voznak, "Hybrid full-duplex/half-duplex relay selection scheme with optimal power under individual power constraints and energy harvesting," Computer Communications, vol. 124, pp. 31-44, June 2018, doi: 10.1016/j.comcom.2018.04.014.

[17] F. K. Ojo and M. F. M. Salleh, "Throughput analysis of a hybridized power-time splitting based relaying protocol for wireless information and power transfer in cooperative networks," IEEE Access, vol. 6, pp. 24137-24147, 2018, doi: 10.1109/ACCESS.2018.2828121.

[18] H-S. Nguyen, D-T. Do, A-H. Bui, and M. Voznak, "Self-powered wireless two-way relaying networks: model and throughput performance with three practical schemes," Wireless Personal Communications: An International Journal, vol. 97, no. 1, pp. 613-631, 2017, doi: 10.1007/s11277-017-4526-3.

[19] F. A. Khan, Z. A. Malik, A. A. Nasir, and M. Masood, "Relay selection \& power allocation for maximizing sumthroughput of a buffered relay network," IEEE Communications Letters, vol. 24, no. 6, pp. 1318-1322, June 2020, doi: 10.1109/LCOMM.2020.2981882.

[20] Y. Feng, V. C. M. Leung, and F. Ji, "Performance study for SWIPT cooperative communication systems in shadowed nakagami fading channels," IEEE Transactions on Wireless Communications, vol. 17, no. 2, pp. 1199-1211, Feb. 2018, doi: 10.1109/TWC.2017.2776933.

[21] J. Zhang, C. Yuen, C. Wen, S. Jin, K. Wong, and H. Zhu, "Large system secrecy rate analysis for SWIPT MIMO wiretap channels," IEEE Transactions on Information Forensics and Security, vol. 11, no. 1, pp. 74-85, Jan. 2016, doi: 10.1109/TIFS.2015.2477050.

[22] S. Y. Seidel, K. Takamizawa, and T. S. Rappaport, "Application of second-order statistics for an indoor radio channel model," IEEE 39th Vehicular Technology Conference, vol. 2, 1989, pp. 888-892, doi: 10.1109/VETEC.1989.40179.

[23] H. Hashemi, "The indoor radio propagation channel," in Proceedings of the IEEE, vol. 81, no. 7, pp. 943-968, July 1993, doi: 10.1109/5.231342.

[24] M. D. Renzo, F. Graziosi, and F. Santucci, "A comprehensive framework for performance analysis of cooperative multi-hop wireless systems over log-normal fading channels," IEEE Transactions on Communications, vol. 58, no. 2, pp. 531-544, February 2010, doi: 10.1109/TCOMM.2010.02.080273.

[25] K. M. Rabie, B. Adebisi, and M. Alouini, "Half-duplex and full-duplex AF and DF relaying with energy-harvesting in log-normal fading," IEEE Transactions on Green Communications and Networking, vol. 1, no. 4, pp. 468-480, Dec. 2017, doi: 10.1109/TGCN.2017.2740258.

[26] A. Salem, K. A. Hamdi, and E. Alsusa, "Physical layer security over correlated log-normal cooperative power line communication channels," IEEE Access, vol. 5, pp. 13909-13921, 2017, doi: 10.1109/ACCESS.2017.2729784.

[27] Y. Liu, R. Xiao, J. Shen, H. Yang, and C. Yan, "Hybrid protocol for wireless energy harvesting network over lognormal fading channel," The Journal of Engineering, vol. 2018, no. 6, pp. 339-341, March 2018, doi: 10.1049/joe.2017.0892.

[28] M. T. Dabiri and S. M. S. Sadough, "Performance analysis of all-optical amplify and forward relaying over lognormal SO channels," Journal of Optical Communications and Networking, vol. 10, no. 2, pp. 79-89, 2018, doi: $10.1364 / \mathrm{JOCN} .10 .000079$. 